\title{
Restrições causadas pela incontinência urinária à vida da mulher
}

\author{
URINARY INCONTINENCE RESTRICTIONS IN WOMEN'S LIFE \\ RESTRICCIONES OCASIONADAS POR LA INCONTINENCIA URINARIA EN LA VIDA DE LA MUJER
}

\author{
Maria Helena Baena de Moraes Lopes', Rosângela Higa²
}

\author{
1 Enfermeira Livre- \\ docente. Professora \\ Associada do Depar- \\ tamento de Enfer- \\ magem da Faculdade \\ de Ciências Médicas \\ da Universidade \\ Estadual de Campinas \\ (FCM/UNICAMP). \\ mhbaena@fam.unicamp.br \\ 2 Enfermeira. Mestre \\ em Enfermagem. \\ Supervisora do \\ Serviço de Enferma- \\ gem da Unidade de \\ Pacientes Externos \\ do Centro de \\ Atenção Integral à \\ Saúde da Mulher \\ (CAISM) - UNICAMP \\ ro-higa@ig.com.br
}

\section{RESUMO}

O objetivo deste estudo foi identificar as restrições causadas pela incontinência urinária (IU) à vida da mulher, considerando-se o tipo de incontinência, e verificar como manejam este problema. Trata-se de uma análise secundária de dados obtidos em estudo anterior, coletados por meio de entrevista com perguntas abertas e fechadas. A amostra foi composta por 164 mulheres com queixa de IU, internadas em clínicas de ginecologia e urologia de dois hospitaisescola da cidade de Campinas, SP. Apresentavam idade entre 25 e 85 anos e 104 (64\%) referiram uma ou mais restrições. Citaram alterações nas atividades sexuais $(40,9 \%)$, sociais $(33,5 \%)$, domésticas $(18,9 \%)$ e ocupacionais $(15,2 \%)$. A incontinência urinária mista e a de urgência forem as que mais afetavam a vida das mulheres. A estratégia mais utilizada para minimizar a IU foi o uso de forro e/ou absorvente higiênico. Concluiu-se que a IU tem implicações negativas no cotidiano dessas mulheres.

\section{DESCRITORES}

Incontinência urinária.

Saúde da mulher.

Diagnóstico de enfermagem.

\section{ABSTRACT}

Our purpose with this study was to verify the restriction of the urinary incontinence (UI) in a woman's daily life, considering the type of incontinence, and find out how women deal with that. It is a secondary analysis of data taken from a previous study that were obtained using open and closed questions. Interviews were held with 164 incontinent inpatients of gynecological and urological clinics of two hospital schools in the city of Campinas, in the State of São Paulo, with ages ranging from 25 to 85 years. Of this universe, 104 (64\%) indicated one or more restrictions regarding their daily life activities, such as altered sexual (40.9\%), social $(33.5 \%)$, domestic (18.9\%) and occupational activities $(15.2 \%)$. Mixed urinary incontinence and urge incontinence were mentioned as that affected the most women's daily life. The disposable pad system was the most utilized strategy in dealing with UI. It was concluded that UI has a negative impact on the daily life of these women.

\section{KEY WORDS}

Urinary incontinence.

Women's health.

Nursing diagnosis.

\section{RESUMEN}

En este estudio se tuvo como objetivo identificar las restricciones causadas por la incontinencia urinaria (IU) en la vida de la mujer, considerándose el tipo de incontinencia, y verificar cómo manejan este problema. Se trata de un análisis secundario de datos obtenidos en un estudio anterior, recolectados por medio de entrevista con preguntas abiertas y cerradas. La muestra estuvo compuesta por 164 mujeres con queja de IU, internadas en clínicas de ginecología y urología de dos hospitales docentes de la ciudad de Campinas, SP. Las mujeres tenían entre 25 y 85 años de edad, habiendo relatado 104 (64\%) de ellas una o más restricciones. Citaron alteraciones en las actividades sexuales $(40,9 \%)$, sociales $(33,5 \%)$, domésticas $(18,9 \%)$ y ocupacionales $(15,2 \%)$. La incontinencia urinaria mixta y la de urgencia fueron las que más afectaban la vida de las mujeres. La estrategia más utilizada para minimizar la IU fue el uso de paños y/o toallas higiénicas. Se concluyó que la IU tiene implicancias negativas en el cotidiano de esas mujeres.

\section{DESCRIPTORES}

Incontinencia urinaria.

Salud de la mujer.

Diagnóstico de enfermería. 


\section{INTRODUÇÃO}

Há alguns anos realizamos um estudo que objetivou desenvolver e avaliar um sistema especialista para diferenciar os diagnósticos de enfermagem relacionados à eliminação urinária alterada ${ }^{(1)}$. Durante a coleta de dados, chamounos a atenção os freqüentes relatos de mulheres com incontinência urinária (IU) que pontuavam diferentes efeitos psicossociais que transcendiam os problemas de saúde.

A International Continence Society (ICS) recentemente alterou a definição de IU, antes considerada como "perda involuntária de urina, que é objetivamente demonstrável e um problema social ou higiênico ${ }^{(2) ",}$ para "perda involuntária de urina que é um problema social ou higiênico"(3), valorizando, com isso, a queixa do paciente. De fato, pesquisas têm mostrado que a IU afeta a qualidade de vida e os problemas higiênicos e sociais têm sido tema de vários estudos, principalmente na literatura médica. Entretanto, observamos que no Brasil são poucas as publicações nacionais de enfermagem sobre o tema. Soma-se a isto a carência de estudos nacionais relativos a índices de prevalência e incidência em diferentes grupos populacionais $^{(4)}$.

A IU é uma condição que afeta a população mundial, principalmente a feminina. Nos Estados Unidos da América, aproximadamente 13 milhões de adultos já vivenciaram algum episódio de IU, entre os quais, 11 milhões (85\%) são mulheres. De acordo com os estudos realizados, a experiência com episódios de perda urinária é uma condição que não prevalece somente em mulheres idosas mas, também, mulheres jovens e na meia-idade. Os problemas urinários não são conseqüências naturais da idade e também não são um problema exclusivo do envelhecimento ${ }^{(5)}$.

Considerando-se a população feminina em geral, a literatura médica aponta os tipos mais comuns de IU, de acordo com os sintomas: incontinência urinária de esforço ("stress incontinence"), que é a perda involuntária de urina associada com atividades físicas que aumentam a pressão intraabdominal; urge-incontinência ("urge incontinence"), definida como perda involuntária de urina associada com um forte desejo de urgência para urinar, e incontinência mista ("mixed incontinence"), quando existe associação dos sintomas de incontinência de esforço e urge-incontinência ${ }^{(2)}$.

A North American Nursing Diagnosis Association (NANDA $)^{(6)}$ apresenta em sua classificação, como diagnósticos de enfermagem, a incontinência urinária de esforço (perda de urina menor que $50 \mathrm{ml}$, que ocorre com o aumento da pressão abdominal) e a incontinência urinária de urgência (perda involuntária de urina que ocorre imediatamente após uma forte sensação de urgência para urinar). Não há um diagnóstico de enfermagem correspondente à incontinência urinária mista, embora estudos tenham apontado e evidenciado a importância deste diagnóstico na prática diária da enfermagem e sugerido a necessidade de sua inclusão na taxonomia dos diagnósticos de enfermagem ${ }^{(7-8)}$.

Alguns fatores influenciam os índices de prevalência da IU na mulher, fazendo com que os resultados não tenham a mesma consistência nos diversos estudos. Estes índices variam de acordo com a metodologia utilizada, isto é, de acordo com as características da população (faixa etária, atividade profissional, presença de doenças crônicas, menopausa, entre outras), da definição do tipo de IU (esforço, urgência ou mista) e da frequiência da perda urinária adotada no estudo (diária, semanal, mensal ou esporádica).

A literatura evidencia prevalência de $14 \%$ a $57 \%$ entre mulheres com queixa de episódios, variando de esporádicos a diários e com idade entre 20 e $89 \operatorname{anos}^{(9-12)}$.

Considerando a prevalência de acordo com os tipos de IU, um estudo com a população feminina dinamarquesa de $30 \mathrm{a}$ 59 anos demonstrou que14, $8 \%$ tinham incontinência urinária por esforço, $8,6 \%$ urge-incontinência e 7,1\% incontinência urinária mista ${ }^{(13)}$. Entre australianas incontinentes de 35 a 64 anos, 55\% tinham incontinência urinária por esforço, $31 \%$ incontinência urinária mista e 10\% urge-incontinência ${ }^{(14)}$. No entanto, em mulheres mais idosas (de 80 a 89 anos) da Islândia, ocorreu inversão da prevalência, isto é, 43\% tinham urge-incontinência, 28\% incontinência urinária mista e $26 \%$ incontinência urinária por esforço ${ }^{(9)}$. No Brasil, um estudo realizado com mulheres incontinentes no climatério e com idade entre 35 e 81 anos, mostrou ser mais freqüente a incontinência urinária por esforço $(30,7 \%)$, seguida pela urge-incontinência $(14,2 \%)$ e incontinência urinária mista $(10 \%)^{(10)}$.

A IU é vista como um problema que afeta mulheres multíparas mais velhas, embora existam evidências de que durante atividades físicas estressantes seja comum entre mulheres jovens, fisicamente ativas, mesmo na ausência de fatores de risco conhecidos.

Entre atletas nulíparas com idade média de 19,9 anos, $28 \%$ reportaram perda urinária durante a prática do espor$\mathrm{te}^{(15)}$, e entre mulheres militares do exército americano, com idade média de 32,2 anos, 30\% relataram experiência com IU durante os exercícios em campo de treinamento, sendo as atividades físicas as indutoras da perda urinária que interferia no desempenho profissional e causava problemas de higiene e embaraço social ${ }^{(16)}$. 
Apenas recentemente o impacto da IU entre homens e mulheres trabalhadores tem sido objeto de estudo. Sendo a população trabalhadora geralmente caracterizada como saudável e considerando as mulheres jovens e profissionalmente ativas, a IU tem apresentado índice de prevalência significativo.

Um estudo sobre seu impacto entre mulheres trabalhadoras, demonstrou que 69,3\% tinham idade inferior a 50 anos e que pode causar fadiga, embaraço, alteração na concentração e estresse emocional durante o expediente de traba1 ho ${ }^{(17)}$. Entre mulheres profissionais da enfermagem, apresentar perda involuntária de urina durante a jornada de trabalho originou sentimentos como o estresse, vergonha, irritação, constrangimento, incômodo e falta de concentração, fatores responsáveis por um impacto negativo no seu desempenho profissional ${ }^{(18)}$.

A IU tem trazido aflição e condições de incapacidade as quais têm causado significativa morbidade entre as mulheres. Entre $15 \%$ a $30 \%$ dos casos afeta a vida social, ocupacional, doméstica, física e sexual das mulheres de todas as idades ${ }^{(19)}$. Seu efeito psicossocial pode ser mais devastador do que as consequiências sobre a saúde, com múltiplos e abrangentes efeitos que influenciam as atividades diárias, a interação social e a autopercepção do estado de saúde ${ }^{(16)}$.

O manejo inadequado do problema como a restrição prolongada de líquidos para reduzir os episódios de IU, pode causar complicações como a infecção urinária, o refluxo e o dano renal ${ }^{(17)}$.

Com base nos relatos citados na literatura sobre o impacto causado pela IU na vida da mulher, foram objetivos do presente estudo identificar em um grupo de mulheres com queixa de incontinência urinária, que participaram do estudo anterior já citado, as restrições causadas pela IU, considerando-se os diferentes tipos, e verificar como as manejam.

\section{SUJEITOS E MÉTODO}

Para este estudo foi realizada análise de entrevistas realizadas com mulheres hospitalizadas em duas unidades de internação: uma ginecológica e outra urológica, de dois hospitais-escola da cidade de Campinas-SP, pertencentes à mesma universidade, que subsidiaram o desenvolvimento e a validação de um sistema especialista para diagnóstico diferencial de alterações na eliminação urinária ${ }^{(1)}$, usando a classificação diagnóstica da NANDA.

O instrumento utilizado foi adaptado da proposta de Carpenito ${ }^{(20)}$ para um "assessment" focalizado, que visa investigar a presença destes diagnósticos de enfermagem. As mulheres que tinham queixa de IU foram entrevistadas e o instrumento utilizado continha perguntas abertas sobre o início da IU, fatores que pioram a incontinência, queixas urinárias, presença de restrições sobre o estilo de vida (sociais, ocupacionais, sexuais, responsabilidade do papel) ${ }^{(20)}$ causadas pela IU, os manejos utilizados para minimizar a perda urinária e um "check-list" com as características definidoras dos diagnósticos de enfermagem. As respostas foram registradas de forma a se constituir uma descrição das queixas (sinais e sintomas) e dos fatores relacionados. Em alguns casos, trechos de fala das mulheres foram registrados de forma literal, porque melhor expressavam o problema.

No presente estudo, foram incluídos apenas os casos de incontinência urinária de esforço (IUE), incontinência urinária de urgência (IUU) e incontinência urinária mista (IUM). Considerou-se como incontinência urinária mista quando a queixa da mulher abrangia características definidoras dos diagnósticos de incontinência urinária de esforço e incontinência urinária de urgência.

O estudo foi aprovado pelo Comitê de Ética em Pesquisa da instituição e as participantes do estudo assinaram um Termo de Consentimento Livre e Esclarecido, de acordo com as normas vigentes ${ }^{(21)}$.

\section{RESULTADOS E DISCUSSÃO}

Foram selecionadas e analisadas as entrevistas de 164 mulheres com queixa de IU. A idade das mulheres variou entre 25 e 85 anos (média de 49,2 anos). Dentre elas, 104 $(63,4 \%)$ referiram uma ou mais restrições nas atividades.

De acordo com os tipos de IU, a maioria $(57,9 \%)$ referiu sintomas de IUM, seguido da IUE $(32,3 \%)$ e da IUU $(9,8 \%)$. Este resultado é compatível com os estudos realizados com a avaliação da IU baseada apenas na queixa clínica, como a realizada na Noruega, envolvendo 3.198 mulheres (2.845 responderam ao questionário), no qual a sintomatologia baseada nas respostas apresentadas pelas pacientes evidenciou índices de 64\% para IUM, 24\% para IUE e 9\% para IUU ${ }^{(22)}$. Em nosso meio, um estudo retrospectivo baseado no atendimento de 114 mulheres observou que a queixa de IUE (perda urinária isolada) foi referida por $36,0 \%$ das pacientes, a IUU (urge-incontinência isolada) por $11,4 \%$ e os sintomas de IUM (sintomas mistos) por $52,6 \%{ }^{(23)}$.

Como se pode observar na Tabela 1, a queixa de IUE ocorreu em média entre mulheres de 44,9 anos, enquanto a IUM e a IUU predominaram nas mulheres com idade média maior. São achados semelhantes ao da literatura que relata ser a IUE a mais comum em mulheres mais jovens, a IUM mais freqüente na idade próxima à menopausa e a IUU, nas mais idosas ${ }^{(11,22)}$. 
Tabela 1 - I dade média, mínima (min) e máxima (max) das mulheres internadas de acordo com o tipo de incontinência ereferência à restrição de atividades causada pela incontinência urinária - Campinas, 2002

I dade

Tipo de Incontinência Urinária

Com restrição $(n=104)$

Sem restrição $(n=60)$

\begin{tabular}{lcccccc} 
& Média & Min & Max & Média & Min & Max \\
\hline Incontinência urinária mista $(n=95)$ & 47,5 & 26 & 71 & 54 & 33 & 85 \\
Incontinência urinária de esforço $(n=53)$ & 44,9 & 25 & 67 & 46,3 & 30 & 68 \\
Incontinência urinária de urgência $(n=16)$ & 56,9 & 34 & 78 & 71,6 & 44 & 80 \\
\hline
\end{tabular}

Considerando-se a queixa de restrições causadas pela IU, os resultados foram diferentes conforme o tipo de IU, pois a maioria das mulheres com queixa de IUM $(78,9 \%) \mathrm{e}$ IUU $(56,3 \%)$ referiram algum tipo de restrição, sugerindo que estas mulheres podem estar sofrendo um maior impacto negativo em sua vida devido à IU do que as mulheres com IUE $(37,7 \%)$ (Tabela 2). Este resultado pode ser ratificado por um estudo realizado na Suécia no qual se constatou que a mulher com IUU ou IUM refere qualidade de vida inferior quando comparada com a mulher que tem IUE ${ }^{(11)}$.
A literatura ressalta que a intensidade com que ocorre a IU (pequena, moderada ou severa), irá influenciar a qualidade de vida da mulher incontinente, ou seja, quanto maior o volume urinário perdido maior será a implicação negativa ${ }^{(24)}$. Considerando que na IUE o volume perdido é geralmente pequeno (gotas) este fato poderia explicar os resultados encontrados.

Tabela 2 - Distribuição das mulheres internadas de acordo com oti po de incontinência urinária e referência à restrição de atividades - Campinas, 2002

\begin{tabular}{|c|c|c|c|c|c|c|}
\hline \multirow{2}{*}{ Tipo de Incontinência Urinária } & \multicolumn{2}{|c|}{ Com restrição $(n=104)$} & \multicolumn{2}{|c|}{ Sem restrição $(n=60)$} & \multicolumn{2}{|c|}{ Total } \\
\hline & $\mathrm{n}$ & $\%$ & $\mathrm{n}$ & $\%$ & $\mathrm{n}$ & $\%$ \\
\hline Incontinência urinária mista & 75 & 78,9 & 20 & 21,1 & 95 & 57,9 \\
\hline I ncontinência urinária de esforço & 20 & 37,7 & 33 & 62,3 & 53 & 32,3 \\
\hline I ncontinência urinária de urgência & 9 & 56,3 & 7 & 43,7 & 16 & 9,8 \\
\hline Total & 104 & 63,4 & 60 & 36,6 & 164 & 100,0 \\
\hline
\end{tabular}

A Tabela 3 mostra que a principal restrição referida pelas mulheres com IUM e IUE foi relativa à atividade sexual, sendo que na IUU foi mais freqüente a restrição nas atividades sociais.

A interferência da IU na vida sexual foi citada por 67 $(40,9 \%)$ mulheres incontinentes. A restrição na atividade sexual era causada por perder urina $(25,6 \%)$, sentir dor durante a relação $(20,7 \%)$, não sentir prazer ou desejo em ter relação $(4,9 \%)$, diminuir ou evitar a atividade sexual $(1,8 \%)$; necessitar interromper a relação para urinar $(1,2 \%)$ e sentir vontade de urinar durante a relação sexual $(1,2 \%)$.

A restrição social foi referida por $55(33,5 \%)$ mulheres. A IU impedia a mulher de sair de casa $(27,4 \%)$, ir a festas e ao clube $(6,7 \%)$, fazer viagens longas $(5,5 \%)$, frequientar a igreja $(4,3 \%)$ e participar de atividades físicas, como: caminhar, correr, jogar e dançar $(3,7 \%)$. Algumas mulheres revelaram que somente saíam de casa quando havia facilidade em utilizar o banheiro $(4,3 \%)$.
As mulheres que deixaram de sair de casa admitiram que sentiam medo ou vergonha de perder urina em público e ficarem molhadas, que cheiravam urina e tinham medo de não encontrar banheiro.

Com relação ao serviço doméstico, $18,9 \%$ (31) se queixaram que a IU causava restrições nas atividades diárias. Estas mulheres necessitavam parar constantemente o serviço para usar o banheiro $(10,4 \%)$ e informaram não realizar atividades que requeriam esforço físico ou carregar peso $(4,3 \%)$.

Quanto às atividades ocupacionais, 15,2\% (25) referiram que a IU interferia no seu desempenho profissional, porque necessitavam interromper o trabalho várias vezes para ir ao banheiro $(8,5 \%)$, evitavam carregar peso $(0,6 \%) \mathrm{e}$ faltavam ao trabalho com frequiência para consultar o médico $(0,6 \%)$. A IU causou também prejuízos financeiros porque foram demitidas ( 2 ou 1,2\%) ou tiveram que pedir demissão $(1$ ou $0,6 \%)$. 
Estes resultados foram consistentes com a literatura. Um estudo que envolveu a população feminina em geral verificou que, entre $15 \%$ e $30 \%$ das vezes, a IU afetava a vida social, ocupacional, doméstica, física e sexual das mulheres de todas as idades ${ }^{(19)}$. Outro estudo realizado com mulheres de 30 a 59 anos, mostrou que estas se abstiveram das atividades sociais $(21,9 \%)$, tiveram alteração na atividade sexual $(3,4 \%)$, faltaram ao trabalho (7\%), deixaram de visitar os amigos $(1,3 \%)$, praticar esportes $(2,6 \%)$ e fazer compras $(18 \%)$ devido à $\mathrm{IU}{ }^{(25)}$.

Entre mulheres trabalhadoras da enfermagem, a IU foi responsável por restrições na atividade ocupacional (18\%), sexual (12\%) e social (1\%). As atividades que demandavam esforços físicos, como carregar peso $(38,7 \%)$ e empurrar macas e cadeiras de rodas (16\%) aumentavam a frequiência da perda urinária durante a jornada de trabalho e, consequentemente, interferiram no desempenho e produtividade destas mulheres ${ }^{(18)}$.

As repercussões no estilo de vida das mulheres com IU são numerosas, normalmente elas sofrem com problemas físicos, econômicos e psicossociais, que interferem no convívio social, profissional, sexual e familiar. Estes problemas contribuem para que ocorram restrições das atividades no lar, perda da confiança em si, vergonha e medo de apresentar um episódio em público, ou na participação dos eventos sociais com amigos e familiares, frustrações e limitações no desenvolvimento do trabalho profissional e disfunção sexual se estiverem molhadas ou perderem urina durante $o$ $\operatorname{orgasmo}^{(24,26-28)}$.

Tabela 3- Atividades afetadas pela incontinência de acordo com otipo deincontinência urinária - Campinas, 2002

\begin{tabular}{|c|c|c|c|c|c|c|c|c|}
\hline \multirow{3}{*}{ Restrições* } & \multicolumn{6}{|c|}{ Tipo de Incontinência Urinária } & \multirow{2}{*}{\multicolumn{2}{|c|}{$\begin{array}{l}\text { Total } \\
(n=164)\end{array}$}} \\
\hline & \multicolumn{2}{|c|}{ IUM ( $n=95)$} & \multicolumn{2}{|c|}{ IUE $(n=53)$} & \multicolumn{2}{|c|}{$\operatorname{IUU}(n=16)$} & & \\
\hline & $\mathrm{n}$ & $\%$ & $\mathrm{n}$ & $\%$ & $\mathrm{n}$ & $\%$ & $\mathrm{n}$ & $\%$ \\
\hline Sexual & 51 & 53,7 & 12 & 22,6 & 4 & 25,0 & 67 & 40,9 \\
\hline Social & 40 & 42,1 & 10 & 18,9 & 5 & 31,3 & 55 & 33,5 \\
\hline Doméstica & 20 & 21,1 & 8 & 15,1 & 3 & 18,8 & 31 & 18,9 \\
\hline Ocupacional & 20 & 21,1 & 4 & 7,5 & 1 & 6,3 & 25 & 15,2 \\
\hline
\end{tabular}

*As mulheres referiram mais de uma atividade afetada pela incontinência, portanto o total não soma $100,0 \%$.

Quanto ao manejo da IU, em $45,1 \%$ dos casos houve necessidade do uso de estratégias, apresentadas na Tabela 4, para tentar minimizar os efeitos da IU. Considerando-se o tipo de incontinência, $54,7 \%$ das mulheres com IUM, 32,1\% daquelas com IUP e $31,2 \%$ dos casos de IUU reportaram uso de uma ou mais estratégias. Portanto, as mulheres com IUM fizeram uso de estratégias com maior freqüência e este resultado está de acordo com o fato de que a maior parte destas mulheres se queixou de alguma restrição.

Considerando que a atividade sexual foi a mais afetada pela IU, algumas mulheres utilizam como estratégia esvaziar a bexiga antes da relação sexual $(7,3 \%)$ ou esvaziar a bexiga antes e após o ato sexual $(2,4 \%)$.

Nesse sentido, foram realizados dois estudos entre mulheres trabalhadoras na Dinamarca. Um deles ${ }^{(17)}$ encontrou achados semelhantes: $30 \%$ utilizavam alguma estratégia para minimizar a IU, $28 \%$ esvaziavam a bexiga em intervalos programados, $18 \%$ limitavam a ingestão hídrica, $14 \%$ não inge- riam cafeína, $13 \%$ levavam roupas extras para troca durante a jornada de trabalho e $11 \%$ usavam perfumes ou desodorantes para minimizar o odor de urina. No outro estudo, os resultados apontavam os seguintes índices: $47,3 \%$ usavam absorventes; $35,2 \%$, desodorante; $25 \%$ limitavam a ingestão de líquidos e $16,2 \%$ trocavam de roupa ${ }^{(29)}$.

Entre mulheres militares, um estudo evidenciou que $68 \%$ usavam medidas preventivas, como manter a bexiga vazia ou usar forro, $19 \%$ usavam a restrição hídrica como precaução contra a incontinência antes das atividades físicas mais árduas e 13\% relataram correr risco de desidratação por restrição hídrica ${ }^{(16)}$.

No estudo realizado com mulheres brasileiras, profissionais de enfermagem, 33\% faziam uso de algum recurso, 25\% utilizavam forro ou absorvente higiênico, 9,3\% faziam restrição hídrica, 5,3\% esvaziavam a bexiga em intervalos programados e $1,3 \%$ usavam perfumes ou desodorantes ${ }^{(15)}$. 
Tabela 4- Estratégias utilizadas para manejo da incontinência de acordo com otipo de incontinência urinária - Campinas, 2002

\begin{tabular}{|c|c|c|c|c|c|c|c|c|}
\hline \multirow{3}{*}{ Estratégia usada* } & \multicolumn{6}{|c|}{ Tipo de Incontinência Urinária } & \multirow{2}{*}{\multicolumn{2}{|c|}{$\begin{array}{l}\text { Total } \\
(n=164)\end{array}$}} \\
\hline & \multicolumn{2}{|c|}{ IUM(n=95) } & \multicolumn{2}{|c|}{ IUE $(n=53)$} & \multicolumn{2}{|c|}{$\operatorname{IUU}(n=16)$} & & \\
\hline & $n$ & $\%$ & $\mathrm{n}$ & $\%$ & $\mathrm{n}$ & $\%$ & $\mathrm{n}$ & $\%$ \\
\hline Usar forro/absorvente & 44 & 46,3 & 13 & 24,5 & 5 & 31,2 & 62 & 37,8 \\
\hline Usar roupa escura & 22 & 2,1 & 2 & 3,8 & 0 & 0 & 24 & 14,6 \\
\hline $\begin{array}{l}\text { Levar e/ou trocar } \\
\text { sempre de roupa }\end{array}$ & 6 & 6,3 & 2 & 3,8 & 0 & 0 & 8 & 4,9 \\
\hline Restrição hídrica & 7 & 7,4 & 0 & 0 & 0 & 0 & 7 & 4,3 \\
\hline $\begin{array}{l}\text { Esvaziar a bexiga em } \\
\text { horário programado }\end{array}$ & 6 & 6,1 & 0 & 0 & 0 & 0 & 6 & 3,7 \\
\hline $\begin{array}{l}\text { Usar sempre saia e } \\
\text { sair somente à noite }\end{array}$ & 1 & 1,1 & 0 & 0 & 0 & 0 & 1 & 0,6 \\
\hline $\begin{array}{l}\text { E svaziar a bexiga } \\
\text { antes de sair de casa }\end{array}$ & 0 & 0 & 1 & 1,9 & 0 & 0 & 1 & 0,6 \\
\hline
\end{tabular}

*As mulheres referiram mais de um tipo de estratégia

Problemas psicológicos e emocionais associados à IU foram citados por $43(26,2 \%)$ mulheres. Sentimentos como vergonha, medo, nervosismo e depressão geralmente estavam relacionados ao forte odor causado pela perda de urina em público.

As mulheres com IUM relataram fazer tratamento para depressão $(5,3 \%)$, ter medo de perder urina durante a relação sexual $(4,2 \%)$, sentir constrangimento e tristeza por causa da incontinência $(2,1 \%)$, ter vergonha dos filhos e culpa por perder urina durante a atividade sexual $(1 \%)$. As mulheres com IUE referiram sentir vergonha do marido por perder urina durante o ato sexual $(3,8 \%)$, enquanto as mulheres com IUU referiram ficar nervosas, chateadas e incomodadas com a incontinência $(6,2 \%)$.

Estudo realizado com mulheres atletas revelou que para $38 \%$ a perda de urina causa sentimento de embaraço, para $22 \%$, ansiedade e para $6 \%$, medo ${ }^{(15)}$.

A mulher incontinente raramente fala sobre o seu problema e, por acreditar não ter cura, muitas sofrem em silêncio. Quando questionadas sobre este assunto, muitas vezes procuram omitir seu problema. É muito comum a mulher dizer: "se eu perco urina, estou velha". Isto é uma razão para que não procure o médico e, amiúde, tem constrangimento de relatar seu problema a um profissional ${ }^{(24)}$.

Isto talvez tenha sido a razão pela qual recentemente a ICS tenha sugerido a inclusão de dados sobre a qualidade de vida na abordagem de indivíduos com $\mathrm{IU}^{(30)}$.

No estudo já citado, que envolveu mulheres trabalhadoras, um terço das que eram incontinentes acreditava que a IU é um problema importante para se resolver, $46 \%$ disseram que este problema necessitava de providências; aproxima- damente $40 \%$ responderam que não sabiam como amenizar o problema e $10 \%$ que não podiam melhorá-lo, $81 \%$ solicitaram mais informações sobre as causas, diagnóstico, tratamento e manejo da $\mathrm{IU}^{(17)}$.

Mulheres com IU freqüentemente são atendidas por urologistas ou ginecologistas, tendendo a aumentar a procura por atendimento no período pós-menopausa, quando há grau elevado de perda urinária involuntária. No entanto, muitas mulheres que apresentam graus leve e moderado de IU não procuram o cuidado médico ${ }^{(31)}$. Nesse sentido, um estudo $^{(11)}$ evidenciou que apenas 5,8\% das mulheres com IU procuram atendimento médico e, dentre estas, $47 \%$ consultam o clínico geral; $57 \%$ o ginecologista e $23 \%$ o urologista.

Outra pesquisa ${ }^{(32)}$ realizada com mulheres incontinentes descreveu que $36 \%$ relataram nunca ter falado sobre sua incontinência com ninguém e somente $23 \%$ consultavam o médico. Entre jovens atletas e profissionais da dança, somente $3,3 \%$ discutiam seu problema com o médico ${ }^{(33)} \mathrm{e}$ entre profissionais da enfermagem, $79 \%$ não procuravam tratamento médico para IU porque apresentavam episódios esporádicos e perda urinária muito pequena $(28,8 \%)$, sendo que outras acreditavam que a IU era um problema comum, sem importância $(22 \%)^{(18)}$.

Embora alguns autores tenham sugerido que o diagnóstico e tratamento inicial devam ser práticas do clínico geral e a primeira investigação da IU, por meio de relatos dos sintomas, deva ser iniciada pela enfermeira em nível de assistência primária, isto geralmente não tem ocorrido ${ }^{(14,31)}$.

No Quadro 1 são apresentados, como exemplo, alguns relatos que evidenciam o quanto a IU afetava a vida destas mulheres. 
"Minha vida virou um inferno, ninguém quer você usando fraldas".

"Desejo morrer, não tenho vida ativa, produtiva e sexual".

"Não tenho mais conforto, tenho cheiro de urina".

"Sentia cheirando urina, como um vaso sanitário".

"Estou sentido melhor após fazer terapia, após a cirurgia pretendo mudar de vida".

"Tinha vontade de urinar durante toda a relação, não via a hora de acabar".

"Sinto que a perda de urina tornou a minha vida social um inferno, eu não tenho mais vida social".

“É muito desagradável, você sempre está úmida, fica um cheiro desagradável, a minha incontinência é insuportável”.

"Eu sinto envergonhada, acho que estou com mais cheiro de urina, não me sinto bem".

“Tenho pavor só em pensar que não vou chegar a tempo ao banheiro e ficar molhada".

"Sou cozinheira e perdi o emprego, ninguém quer uma pessoa cozinhando ou servindo a comida cheirando urina".

\section{CONCLUSÕES}

No grupo de mulheres estudado a IU foi causa de restrições nas atividades sexuais, sociais, domésticas e ocupacionais. A IUM e a IUU são as que mais afetam a vida das mulheres. A maioria das mulheres com IUM $(78,9 \%)$ referiu uma ou mais restrições, sendo afetada principalmente a atividade sexual $(53,4 \%)$. Entre as estratégias utilizadas para manejo da IU, a mais comum foi o uso do forro/absorvente higiênico.

Observou-se que a IU provoca sentimentos de baixa auto-estima na mulher e interfere na sua vida sexual, restringe o contato social, interfere nas tarefas domésticas e no trabalho. Além disso, acarreta problemas econômicos devido a gastos com absorventes e, por vezes, ao impedir ou dificultar o trabalho remunerado fora de casa.

Com o aumento da perspectiva de vida das populações em geral, haverá um número crescente de idosos e, consequientemente, o número de casos de IU pode au-

\section{REFERÊNCIAS}

(1) Lopes MHBM, Higa R. Desenvolvimento de um sistema especialista para identificação de diagnóstico de enfermagem relacionado com a eliminação urinária. Rev Bras Enferm 2005; 58(1):27-32.

(2) Abrams P, Blaivas JG, Stanton SL, Andersen JT. The standardization of terminology of lower urinary tract function. Scand J Urol Nephrol. 1988;22(Suppl 114):5-19.

(3) Abrams P, Cardoso L, Fall M, Griffiths D, Rosier P, Ulmsten $\mathrm{U}$, et al. The standardisation of terminology of lower urinary tract function: report from the standardisation sub-committee of the International Continence Society. Urology. 2003;61(1):37-49.

(4) Silva APM, Santos VLCG. Prevalência da incontinência urinária em adultos e idosos hospitalizados. Rev Esc Enferm USP. 2005;39(1):36-45. mentar. Muitos deles não serão diagnosticados pela falta de busca de tratamento por parte da clientela e pelo despreparo dos profissionais de saúde na abordagem, diagnóstico e tratamento.

O problema da IU tem sido subestimado e não tem recebido adequada atenção. Embora muitas mulheres a considerem uma condição normal e resultado do processo de envelhecimento, a IU tem causado na mulher depressão e isolamento social.

Em se tratando da clientela feminina, além do tratamento clínico ou cirúrgico, a orientação quanto ao manejo do problema, de forma mais adequada em cada caso, deve ser igualmente considerada e valorizada.

A enfermeira tem um papel importante junto dessas mulheres na prevenção, no diagnóstico, por meio da queixa clínica, e na orientação do manejo adequado, evitando que condutas inadequadas, como a restrição prolongada de líquidos e a micção não freqüente, sejam tomadas, o que pode causar complicações e danos à saúde destas mulheres.
(5) Agency for Health Care Policy and Research (AHCPR). Overview: urinary incontinence in adults clinical practice guideline update. [online] Rockville, MD. March 1996. Available from: http://www.ahcpr.gov/clinic/uiovervw.htm (02 ago. 2003).

(6) North American Nursing Diagnosis Association (NANDA). Diagnósticos de enfermagem da NANDA: definições e classificações 2003 - 2004. Tradução de Cristina Correa. Porto Alegre: Artmed, 2005.

(7) Woodtli A. Mixed incontinence: a new nursing diagnosis. Nurs Diagn 1995;6(4):135-42.

(8) Lopes MHBM, Higa R. Mixed incontinence in women: a new nursing diagnosis? Int J Nurs Terminol Classif. 2003;14 (suppl 14):49. 
(9) Bjornsdóttir LT, Geirsson RT, Jónsson PV. Urinary incontinence and urinary tract infections in octogenarian women. Acta Obstet Gynecol Scand. 1998;77(1):105-9.

(10) Guarisi T, Pinto-Neto AM, Pedro AO, Costa-Paiva LH, Faundes A. Sintomas urinários e genitais em mulheres climatéricas. J Bras Ginecol. 1998; 108(4):125-30.

(11) Simeonova Z, Milsom I, Kullendorff AM, Molander U, Bengtsson $\mathrm{C}$. The prevalence of urinary incontinence and its influence on the quality of life in women from an urban Swedish population. Acta Obstet Gynecol Scand. 1999; 78(6):546-51.

(12) Sampselle CM, Harlow SD, Skurnick JS, Brubaker L, Bondarenko I. Urinary incontinence predictors and life impact in ethically diverse perimenopausal women. Obstetr Gynecol. 2002;100(6):1230-8.

(13) Foldspang A, Mommsen S, Lam GW, Elving L. Parity as a correlate of adult female urinary incontinence prevalence. J Epidemiol Community Health. 1992;46(6):595-600.

(14) O'Brien J, Austin M, Seth P, O'Boyle P. Urinary incontinence: prevalence, need for treatment, and effectiveness of intervention by nurse. Br Med J. 1991;303(23):1308-12.

(15) Nygaard IE, Thompson FL, Svengalis SL, Albrigth JP. Urinary incontinence in elite nulliparous athletes. Obstetr Gynecol. 1994;84(2):183-7.

(16) Davis G, Sherman R, Wong MF, McClure G, Perez R, Hibbert M. Urinary incontinence among female soldiers. Mil Med.1999; 164(3):182-7.

(17) Fitzgerald S, Palmer MH, Berry SJ, Hart K. Urinary incontinence. Impact on working women. AAOHN J. 2000;8(3):112-8.

(18) Higa R. Incontinência urinária: problema ocupacional entre profissionais de enfermagem. [dissertação] Campinas (SP): Faculdade de Ciências Médicas da UNICAMP; 2004.

(19) Thomas TM, Plymat KR, Blannin J, Mead TW. Prevalence of urinary incontinence. Br Med J. 1980; 281(6250):1243-5.

(20) Carpenito LJ. Diagnóstico de enfermagem: aplicação à prática clínica. Tradução de Ana Thorell. $6^{\mathrm{a}}$ ed. Porto Alegre: Artes Médicas, 1997. p. 636-54.

(21) Conselho Nacional de Saúde. Resolução n. 196, de 10 de outubro de 1996. diretrizes e normas regulamentadoras sobre pesquisa envolvendo seres humanos. Bioética, Brasília, v. 4, n. 2, p. 15-25, 1996. Suplemento.

(22) Indrrekvan S, Fosse OAK, Hunskaar SA. Norwegian national cohort of 3198 women treated with home-managed electrical stimulation for urinary incontinence. Scand J Urol Nephol. 2001;35(1):26-31.

(23) Feldner PC, Bezerra RLPS, Girão MJBC, Castro RA, Sartori MGF, Bacarat EC, et al. Valor da queixa clínica e exame físico no diagnóstico da incontinência urinária. Rev Bras Ginecol Obstet. 2002;24(2):87-91.
(24) Butler RN, Maby JI, Montella JM, Yong GGPH. Urinary incontinence: keys to diagnosis of the older woman. Geriatrics. 1999;54(10):29-30.

(25) Foldspang A, Mommsen S. The International Continence Society (ICS) incontinence definition: is the social and hygienic aspect appropriate for etiologic research? J Clin Epidemiol. 1997;50(9):1055-60.

(26) Milsom I, Ekelund P, Molander U, Arvdsson L, Areskoug B. The influence of age, parity, oral contraception, hysterectomy and menopause on the prevalence of urinary incontinence in women. J Urol. 1993;149(6):1459-62.

(27) Gallo ML, Fallon PJ, Staskin DR. Urinary incontinence: steps to evaluation, diagnosis, and treatment. Nurse Pract. 1997; 22(2):21-4.

(28) Doren M. Urogenital aging: creation of improved awareness. Am J Obstet Gynecol. 1998; 178(5):S254-6.

(29) Fitzgerald S, Palmer MH, Kikland VL, Robinson L. The impact of urinary incontinence in working women: a study in a production facility. Women Health. 2002;35(1):1-16.

(30) Kelleher CJ, Cardozo LD, Khullar V, Salvatore S. A new questionnaire to assess the quality of life of urinary incontinent women. Br J Obstet Gynaecol. 1997; 104 (12): 1374-9.

(31) Krissovick M. The financial side of continence promotion. Geriatr Nurs. 1998;19(2):91-4.

(32) Reymer J, Hunskaar S. Why do only a minority of perimenopausal women with urinary incontinence consult a doctor? Scand J Prim Health Care. 1994; 12(3):180-3.

(33) Thyssen L, Clevin L, Olesem, S.; Lose G. Urinary incontinence in elite female athletes and dancers. Int Urogynecol J Pelvic Floor Dysfunct. 2002;13(1):13-5. 